\title{
Joint 2D Hybrid MIMO Phased-Array and Nested Array for Increased Apertures
}

\author{
Shuaiqi Liu ${ }^{+}$, Buhong Wang, Shuai Cao, Xinbo Liu \\ Information and Navigation College, Air Force Engineering University, Xi'an
}

\begin{abstract}
In this paper, a receive array design method based on 2D nested array for two dimensional Hybrid MIMO phased-array radar is proposed. It can offers more apertures by forming difference coarray, which will defeat the apertures sacrifice brought by the two dimensional Hybrid MIMO phased-array radar as compared to the traditional MIMO radar. Spatial smoothing will also used to form the subspace based DOA estimation like Unitary ESPRIT. Numerical simulation will validate the proposed approach.
\end{abstract}

Keywords: 2D Hybrid MIMO phased-array radar, 2D Nested array, direction of arrival (DOA) estimation, Unitary ESPRIT.

\section{Introduction}

Multiple-input multiple-output (MIMO) radar superiorities due to construct virtual aperture that is larger than the physical array [1]. Hence, it offers significant performance improvements such as higher angular resolution, higher sensitivity to detect targets, increasing number of detectable targets [2] and combating the signal scintillation [3]. However, MIMO radar sacrifices transmit coherent processing gain as compared to phased-array radar [4]. This can lead to signal-to-noise radio (SNR) loss in the receive array which then influence DOA estimation accuracy when practical application. Focusing on the MIMO radar with collocated arrays, Hybrid MIMO phased-array radar is proposed to add transmits coherent processing gain in the traditional MIMO radar [5]. The essence of this technique is dividing the transmit array into several subarrays, then one waveform can be transmitted coherently from each element of the same subarray, different orthogonal waveforms are transmitted from different subarrays. Combining the advantages of MIMO radar and phased radar, Hybrid MIMO Phased-array radar can work in a more flexible environment and improve the performance of DOA estimation.

However, the Hybrid MIMO Phased-array radar sacrifices some apertures due to the transmit array dividing as compared to the traditional MIMO radar. For example, when the transmit element is $\mathrm{M}$, which is assumed equal to the receive element. And the subarray is $\mathrm{K}$, which satisfy $\mathrm{K}<=\mathrm{M}$. The virtual apertures is MM in MIMO radar as KM in Hybrid MIMO Phased-array radar, which will impacts the DOA estimation when there are more targets. In order to save this problem, some algorithms have been developed for increasing apertures of the Hybrid MIMO Phased-array radar. The overlapped transmit dividing method proposed in [6] enhance elements to work in different subarrays at the same time, which dramatic increases the virtual apertures. But it is not easy to be achieved in actual environment. The joint phased-array and nested array model proposed in [7] provides a new way for us, but it only used in liner array. The 2D array is more practically than the liner one in many aspects. So how to improve the apertures of the $2 \mathrm{D}$ Hybrid MIMO phased-array radar is a problem which is waiting to be solved.

According to the discussion, we aim at improving apertures by applying 2D Nested array[8] in receive array of the 2D Hybrid MIMO phased-array radar. Which is based on the difference coarray processing and

\footnotetext{
+ Corresponding author. Tel.: 13002959756

E-mail address: 452156104@qq.com.
} 
spatial smoothing. The main contribution of our work can be summarized as follows: (1) apply 2D nested array in the receive array of the 2D Hybrid MIMO phased-array radar. (2) Give signal model of the joint 2D Hybrid MIMO phased-array and 2D nested array. (3) Spatial smoothing is presented resolve the coherent noise problem caused by the difference coarray.

The paper is organized as follow. 2D nested array model is given in section 2. Two dimensional Hybrid MIMO phased-array radar transmit signal model is briefly introduced in section 3, while receive signal model is in section 4 . In section 5 , simulation examples for the proposed method are drawn.

\section{2D Nested Array}

In order to use 2D estimation algorithm, the element on the array must be contiguous, that is to say, have on halves. Hence, we refer to the configuration II in [9]. Consider a 2D nested array consists of two 2D arrays: sparse and dense array, whose location are demonstrated by $2 \times 2$ nonsingular lattice $N^{s}$ and $N^{d}$, where $\boldsymbol{N}^{s}=\boldsymbol{N}^{d} \boldsymbol{P}$. Here $\boldsymbol{P}=\left[\begin{array}{ll}\lambda_{1} & \\ & \lambda_{2}\end{array}\right]$ is an integer matrix. The number of elements on the two array are integer $N^{s}$ and $N^{d}$.

(a) A dense array with $N^{d}=\operatorname{det}(\boldsymbol{P})$ elements on lattice $N^{d}$, and the elements locations are given by $N^{d} \boldsymbol{n}^{d}$, which can be represented as

$$
\begin{array}{cc}
N^{d}[m, n]^{\mathrm{T}} & -\frac{\left(\lambda_{1}-1\right)}{2} \leq m \leq \frac{\left(\lambda_{1}-1\right)}{2} \\
-\lambda_{2}+1 \leq n \leq 0
\end{array}
$$

(b) Given integers $N^{s 1}$ and $N^{s 2}$, where $N^{s}=\left(2 N^{s 1}+1\right) N^{s 2}-1$.We place a total of $N^{s}$ elements on sparse array at locations are given as follows

$$
\begin{array}{cc} 
& -\boldsymbol{N}^{s l} \leq m \leq \boldsymbol{N}^{s l} \\
& 0 \leq n \leq \boldsymbol{N}^{s 2}-1,[m, n]^{\mathrm{T}} \neq \mathbf{0}
\end{array}
$$

For example, a two level nested array is shown in Fig.1, the dense lattice is $\boldsymbol{N}^{d}=\left[\begin{array}{ll}1 & 0 \\ 0 & 1\end{array}\right]$, and $\boldsymbol{P}=\left[\begin{array}{ll}3 & 0 \\ 0 & 3\end{array}\right]$. Hence the dense array has 9 elements, and the sparse lattice is $N^{s}=\left[\begin{array}{ll}3 & 0 \\ 0 & 3\end{array}\right]$. Here we assume $N^{s 1}=1, N^{s 2}=6$, so the sparse array has 14 elements. Its corresponding difference coarray is depicted in Fig. 2. It can be seen from Fig. 2 that the elements of the dense and sparse array are placed on opposite sides with respect to the origin.

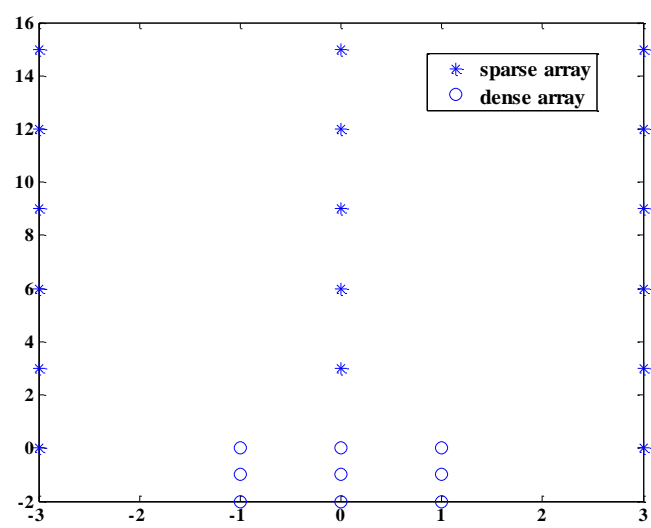

Fig. 1: 2D nested array of configuration II.

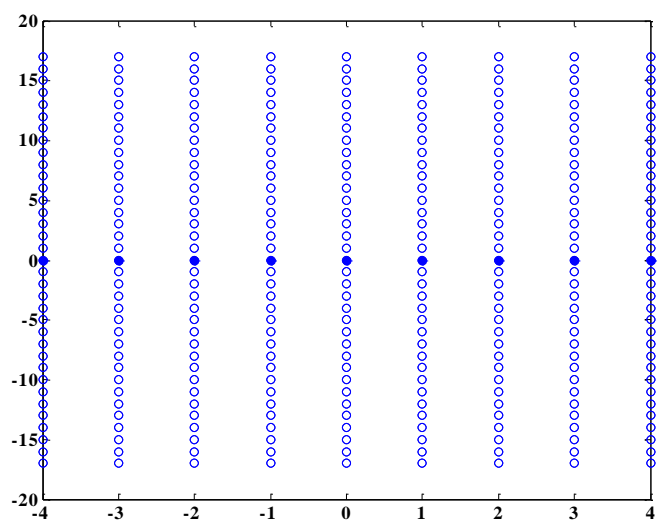

Fig. 2: Difference coarray

\section{Transmit Signal Model}

Consider a two dimensional Hybrid MIMO phased-array radar system with $M_{t} \times N_{t}$ antennas uniform rectangular array (URA) in transmit and $D_{r}$ antennas in receive array. The elements on any given column in the transmit array are assumed to be equally spaced. Where $d_{m}$ and $d_{n}$ are the distances between the adjacent antennas at each column and at each row respectively. Let the steering vector of the each transmit subarray represented as

$$
\boldsymbol{a}_{k}(\theta, \phi)=\operatorname{vec}\left(\boldsymbol{Z}_{k} \odot\left[\boldsymbol{\mu}(\theta, \phi) \boldsymbol{v}^{\mathrm{T}}(\theta, \phi)\right]\right)
$$


where $\boldsymbol{Z}_{k}$ is an matrix of ones and zeros where the $m n^{\text {th }}$ entry equals zero if the $m n^{\text {th }}$ element of the array is absent, vec(.) stands for the operator that stacks the columns of a matrix in one column vector, $\odot$ stands for the Hadamard product, $(\cdot)^{\mathrm{T}}$ denotes the transpose, $\theta$ and $\phi$ denote the elevation and azimuth angles, respectively. $\boldsymbol{\mu}(\theta, \phi)$ and $\boldsymbol{v}(\theta, \phi)$ are defined as follows

$$
\boldsymbol{\mu}(\theta, \phi)=\left[1, \mathrm{e}^{\mathrm{j} 2 \pi d_{m} \sin (\theta) \cos (\phi)}, \cdots, \mathrm{e}^{\mathrm{j} 2 \pi\left(M_{t}-1\right) d_{m} \sin (\theta) \cos (\phi)}\right]^{\mathrm{T}}, \boldsymbol{v}(\theta, \phi)=\left[1, \mathrm{e}^{\mathrm{j} 2 \pi d_{n} \sin (\theta) \sin (\phi)}, \cdots, \mathrm{e}^{\mathrm{j} 2 \pi\left(N_{t}-1\right) d_{n} \sin (\theta) \sin (\phi)}\right]^{\mathrm{T}}
$$

Let $\varphi(t)=\left[\varphi_{1}(t), \cdots, \varphi_{K}(t)\right]^{\mathrm{T}}$ be the vector of predesigned independent waveforms which satisfy the orthogonality condition $\int_{\mathrm{T}_{0}} \boldsymbol{\varphi}(t) \varphi(t)^{\mathrm{H}}=\boldsymbol{I}_{K}, \boldsymbol{I}_{K}$ is the identity matrix of size $K \times K$. Assume a target at direction of $\left(\theta_{t}, \phi_{t}\right)$, the signal reflected by the target is

$$
r\left(t, \theta_{t}, \phi_{t}\right)=\sqrt{M_{t} N_{t}} / K \beta_{t} \sum_{k=1}^{K} \boldsymbol{w}_{k}{ }^{\mathrm{H}} \boldsymbol{a}_{k}\left(\theta_{t}, \phi_{t}\right) \mathrm{e}^{-\mathrm{j} \tau_{k}\left(\theta_{n}, \phi_{t}\right)} \varphi_{k}(t)
$$

where $\beta_{t}$ is the reflection coefficient associated with the target. $w_{k}$ is the transmit weight vector used to form the $k^{\text {th }}$ transmit beam. $\tau_{k}\left(\theta_{t}, \phi_{t}\right)$ is the time required for the signal to cover from the distance between the first element of the $k^{\text {th }}$ subarray.

In addition to the desired target, we also assume there $H$ interfering targets at $\left(\theta_{i}, \phi_{i}\right)$. The received data vector at receive array can be modeled as

$$
\boldsymbol{x}(t)=r\left(t, \theta_{t}, \phi_{t}\right) \boldsymbol{b}\left(\theta_{t}, \phi_{t}\right)+\sum_{i=1}^{L} r\left(t, \theta_{i}, \phi_{i}\right) \boldsymbol{b}\left(\theta_{i}, \phi_{i}\right)+\boldsymbol{n}(t)
$$

where $\boldsymbol{b}(\theta, \phi)$ is the $D_{r} \times 1$ receive steering vector and $\boldsymbol{n}(t)$ is the noise that is supposed to have zero mean. By applying matched filtering to the received data vector for each of the orthogonal waveforms $\varphi_{k}(t), k=1,2 \cdots K$. The $K D_{r} \times 1$ virtual receive data is

$$
\boldsymbol{y}=\int_{T_{0}} \boldsymbol{x}(t) \boldsymbol{\varphi}_{k}^{*}(t) d t=\sqrt{\frac{M_{t} N_{t}}{K}} \beta_{t} \boldsymbol{u}\left(\theta_{t}, \phi_{t}\right)+\sum_{i=1}^{H} \sqrt{\frac{M_{t} N_{t}}{K}} \beta_{i} \boldsymbol{u}\left(\theta_{i}, \phi_{i}\right)+\hat{n}
$$

where $\hat{n}=\int_{T_{0}} \boldsymbol{n}(t) \boldsymbol{\varphi}_{k}{ }^{*}(t) d t$ is the $K D_{r} \times 1$ noise term whose covariance is $\sigma_{n}^{2} \boldsymbol{I}_{K D_{\mathrm{D}} \times 1}$.

\section{Receive Signal model based on 2D Nested Array}

We rewrite (6) as a more general equation

$$
\boldsymbol{y}=\sum_{d=1}^{D} \sqrt{\frac{M_{t} N_{t}}{K}} \beta_{d} \boldsymbol{u}\left(\theta_{d}, \phi_{d}\right)+\hat{n}
$$

Where $D=L+1$ is the number of sources including target and interferences. Then multiply both sides of (7) by $\sqrt{\frac{K}{M_{t} N_{t}}}$,we have

$$
\tilde{\boldsymbol{y}}=\boldsymbol{y} \sqrt{\frac{K}{M_{t} N_{t}}}=\sum_{d=1}^{D} \beta_{d} \boldsymbol{u}\left(\theta_{d}, \phi_{d}\right)+\sqrt{\frac{K}{M_{t} N_{t}}} \hat{n}=\boldsymbol{U} \boldsymbol{\beta}+\sqrt{\frac{K}{M_{t} N_{t}}} \hat{n}
$$

where $\boldsymbol{U}$ denotes the virtual array manifold matrix and $\boldsymbol{\beta}=\left[\begin{array}{llll}\beta_{1}\left(\theta_{1}, \phi_{1}\right) & \beta_{2}\left(\theta_{2}, \phi_{2}\right) & \cdots & \beta_{D}\left(\theta_{D}, \phi_{D}\right)\end{array}\right]^{\mathrm{T}}$ denotes the reflection coefficients matrix. According to the difference coarray processing algorithm, we can get the autocorrelation matrix of $\tilde{y}$ as

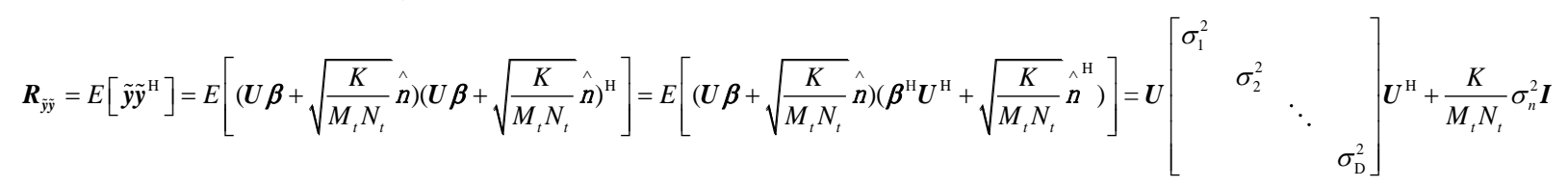

where $\sigma_{n}^{2}$ is the noise variance. And then we vectorize $\boldsymbol{R}_{\tilde{y} \tilde{y}}$ as the following vector

$$
\hat{\boldsymbol{y}}=\operatorname{vec}\left(\boldsymbol{R}_{\tilde{\boldsymbol{y}}}\right)=\operatorname{vec}\left[\sum_{d=1}^{D} \sigma_{d}^{2} \boldsymbol{u}\left(\theta_{d}, \phi_{d}\right) \boldsymbol{u}\left(\theta_{d}, \phi_{d}\right)^{\mathrm{H}}\right]+\frac{K}{M_{t} N_{t}} \sigma_{n}^{2} \tilde{\boldsymbol{I}}_{n}=\left(\boldsymbol{U}^{*} \oplus \boldsymbol{U}\right) \boldsymbol{P}+\frac{K}{M_{t} N_{t}} \sigma_{n}^{2} \overrightarrow{\boldsymbol{I}}_{n}
$$

where the sources power vector is $\boldsymbol{P}=\left[\sigma_{1}^{2}, \sigma_{2}^{2} \cdots \sigma_{\mathrm{D}}^{2}\right]$ and $\overrightarrow{\boldsymbol{I}}_{n}=\left[e_{1}^{\mathrm{T}}, e_{2}^{\mathrm{T}} \cdots e_{K}^{\mathrm{T}}\right]^{\mathrm{T}}$ whose element $\boldsymbol{e}_{i}$ stands for a $\left(\left(2 N_{1}^{s}+1\right) N_{2}^{s}+\operatorname{det}(\boldsymbol{P})-1\right)^{2} \times 1$ column vector of all zeros except a 1 at the $i$ th position. And the symbol $\oplus$ is used to denote Khatri Rao (KR) product. We denote $\boldsymbol{C}=\boldsymbol{U}^{*} \oplus \boldsymbol{U}$, and the matrix $\boldsymbol{C}$ can be represented as 


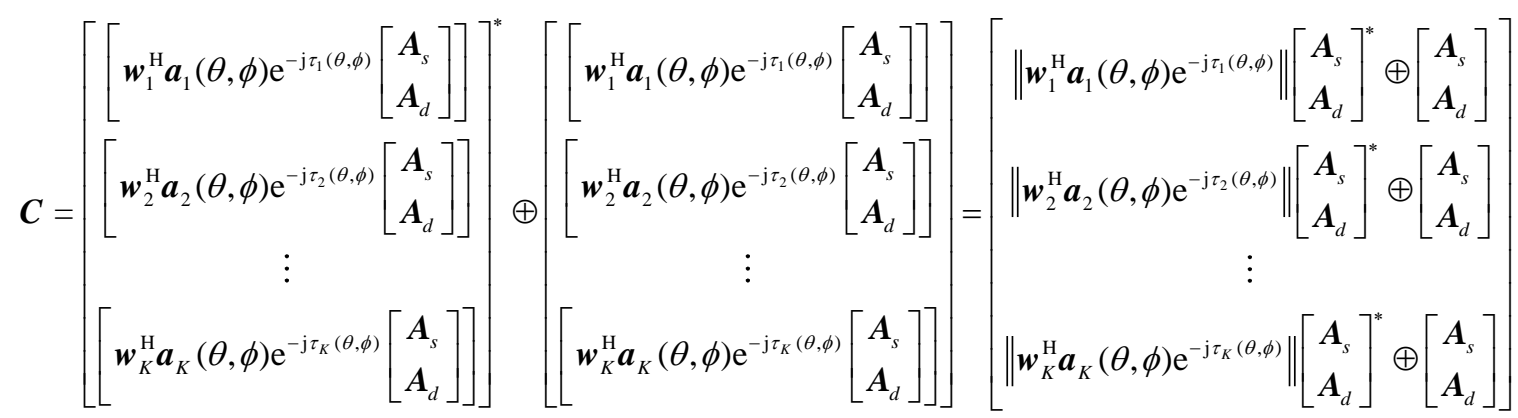

It can be verified that the rows of $\left[\begin{array}{l}\boldsymbol{A}_{s} \\ \boldsymbol{A}_{d}\end{array}\right]^{*} \oplus\left[\begin{array}{l}\boldsymbol{A}_{s} \\ \boldsymbol{A}_{d}\end{array}\right]$ contain all rows of

$$
\begin{array}{lc}
a_{i, k}=\left[\mathrm{e}^{\mathrm{j} \frac{2 \pi}{\lambda} u_{1}^{\mathrm{T}}\left(\boldsymbol{N}^{s} \boldsymbol{n}_{i}^{s}-N^{d} \boldsymbol{n}_{k}^{d}\right)}, \cdots, \mathrm{e}^{\mathrm{j} \frac{2 \pi}{\lambda} u_{D}^{\mathrm{T}}\left(N^{s} \boldsymbol{n}_{i}^{s}-N^{d} \boldsymbol{n}_{k}^{d}\right)}\right] & i=1, \cdots,\left(2 N_{1}^{s}+1\right) N_{2}^{s}-1 . k=1, \cdots, \operatorname{det}(\boldsymbol{P}) \\
a_{i, k}^{d}=\left[\mathrm{e}^{\mathrm{j} \frac{2 \pi}{\lambda} u_{1}^{\mathrm{T}}\left(N^{d} n_{i}^{d}-N^{d} \boldsymbol{n}_{k}^{d}\right)}, \cdots, \mathrm{e}^{\mathrm{j} \frac{2 \pi}{\lambda} u_{D}^{\mathrm{T}}\left(N^{d} n_{i}^{d}-N^{d} n_{k}^{d}\right)}\right] & i, k=1, \cdots, \operatorname{det}(\boldsymbol{P}) \\
a_{i, k}^{s}=\left[\mathrm{e}^{\mathrm{j} \frac{2 \pi}{\lambda} u_{1}^{\mathrm{T}}\left(N^{s} \boldsymbol{n}_{i}^{s}-N^{s} \boldsymbol{n}_{k}^{s}\right)}, \cdots, \mathrm{e}^{\mathrm{j} \frac{2 \pi}{\lambda} u_{D}^{\mathrm{T}}\left(N^{s} \boldsymbol{n}_{i}^{s}-N^{s} \boldsymbol{n}_{k}^{s}\right)}\right] & i, k=1, \cdots,\left(2 N_{1}^{s}+1\right) N_{2}^{s}-1
\end{array}
$$

where the total number of the three kind of rows is $\left(\left(2 N_{1}^{s}+1\right) N_{2}^{s}+\operatorname{det}(\boldsymbol{P})-1\right)^{2}$.In fact, there are many repeat rows contained in the three kind of rows which need to be removed. Hence a new matrix $\boldsymbol{A}_{d i f f}$ with distinct rows and resort the rows corresponding to the element locations in the difference coarray of 2D nested array is constructed. Then the matrix $\hat{y}$ can be represented as

$$
\hat{\boldsymbol{y}}=\left[\begin{array}{c}
\left\|\boldsymbol{w}_{1}^{\mathrm{H}} \boldsymbol{a}_{1}(\theta, \phi) \mathrm{e}^{-\mathrm{j} \tau_{1}(\theta, \phi)}\right\| \boldsymbol{A}_{\text {diff }} \\
\left\|\boldsymbol{w}_{2}^{\mathrm{H}} \boldsymbol{a}_{2}(\theta, \phi) \mathrm{e}^{-\mathrm{j} \tau_{2}(\theta, \phi)}\right\| \boldsymbol{A}_{\text {diff }} \\
\vdots \\
\left\|\boldsymbol{w}_{K}^{\mathrm{H}} \boldsymbol{a}_{K}(\theta, \phi) \mathrm{e}^{-\mathrm{j} \tau_{K}(\theta, \phi)}\right\| \boldsymbol{A}_{\text {diff }}
\end{array}\right] \boldsymbol{P}+\frac{K}{M_{t} N_{t}} \sigma_{n}^{2} \tilde{\mathbf{e}}
$$

where $\boldsymbol{A}_{\text {diff }}$ is an $M L \times D$ matrix and $\tilde{\mathbf{e}}$ is a deterministic vector, and $M=\left(2 N^{s 1}+1\right) \lambda_{1}, L=2 N^{s 2} \lambda_{2}-1$. However, it is worth note that the source signal vector $\boldsymbol{P}$ with actual signal power behaves like fully coherent sources. In order to solve this this problem, [9] adapted the 2D smoothing technique to enhance the rank of the positive semidefinite matrix. By dividing the coarray into $(M+1)(L+1) / 4$ overlapping identical subarrays of size $((M+1) / 2) \times((L+1) / 2)$, the $2 \mathrm{D}$ spatial smoothing on the coherent signal model of $(14)$ is expressed as

$$
\boldsymbol{R}_{\text {coarray }}=\frac{1}{\sqrt{(M+1)(L+1) / 4}}\left(\boldsymbol{A}_{0,0} \boldsymbol{R}_{s s} \boldsymbol{A}_{0,0}^{\mathrm{H}}+\sigma_{n}^{2} \boldsymbol{I}_{\frac{(M+1)(L+1)}{4}}\right)
$$

where $\boldsymbol{A}_{0,0}$ denotes the first subarray manifold matrix in the difference coarray of the $2 \mathrm{D}$ nested array. The matrix $\boldsymbol{R}_{s s}$ contains signal powers. By applying subspace based method like Unitary ESPRIT which has already researched in [10], it is capable to estimate 2D DOA of $O\left(N_{s} N_{d}\right)$ signals using only $O\left(N_{s}+N_{d}\right)$ physical sensors, which is not possible for traditional arrays.

\section{Simulation Results}

In our simulations, we assume a $5 \times 5$ URA with $d_{m}=d_{n}=\lambda / 2$, where $\lambda$ is the wavelength. The target spatial range is defined by $\Theta=[-60,-20], \Phi=[20,60]$. The $2 \mathrm{D}$ transmit array is divided into $K=2$ subarrays that are overlapped and each of them consists of 24 antennas. The noise is complex Gaussian with zero mean. The overlapped transmit array has been shown in[10]. The 2D nested array has been introduced in Section 2.1.

We first assume 10 targets to be estimated, whose location can be described as $\left(\theta_{i}, \phi_{i}\right)=(-90+18 i, 18 i)$, $i=1 \cdots 10$.A receive array with 23 physical elements is used to compare with the $18 \times 5$ virtual difference coarray. The RMSE is computed by averaging over 500 Monte Carlo runs. It can be seen from Fig. 3 that the proposed nested receive array enjoys significant performance compared to the traditional physical array in RMSE.

Then, we assume more targets $L=30(30>23=$ number of physical elements in the array), whose locations are chosen by $\left(\theta_{i}, \phi_{i}\right)=(-90+6 i, 6 i) \quad i=1 \cdots 30$.For the given number of targets, the estimation methods can not be used on the physical array. By using difference coarray processing in Section 2.3, we can estimate more targets using the virtual 
array. Fig. 4 show the estimation obtained from different values of snaphsots $\mathrm{T}=100$ and $\mathrm{T}=1000$, which all achieved by averaging over 500 Monte Carlo runs.

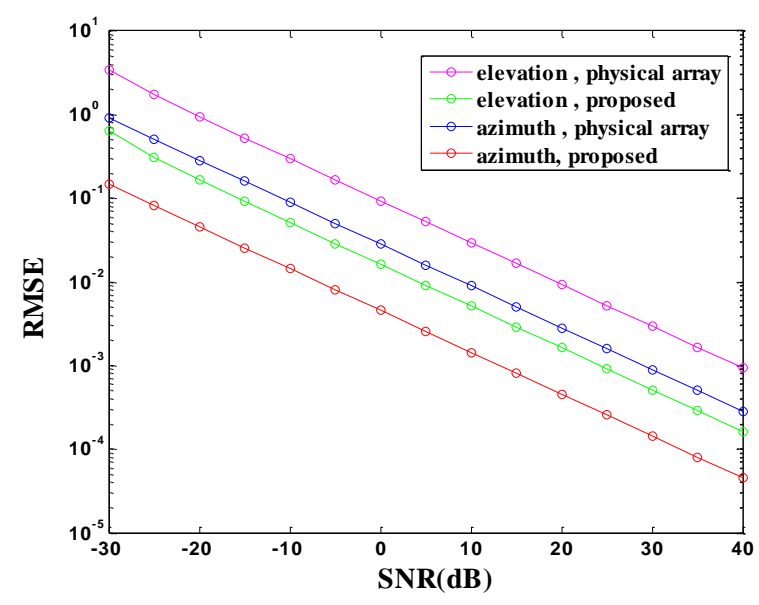

Fig. 3: 2D nested array of configuration II.

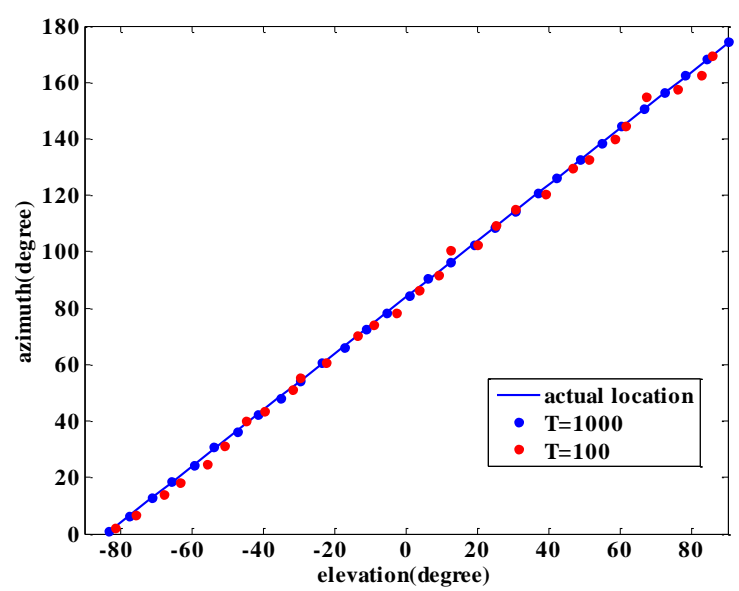

Fig. 4: Difference coarray

\section{Acknowledgements}

This work was supported by the National Natural Science Fund of China (61671465).

\section{References}

[1] J. Li and P. Stoica, MIMO Radar Signal Processing. New Jersy: Wiley, 2009.

[2] J. Li and P. Stoica, MIMO radar with collocated antennas. IEEE Signal Processing Magaz, 2007, 25(1):106-114.

[3] A. Haimovich, R. Blum, and L. Cimini, MIMO Radar with Widely Separated Antennas, IEEE Signal Processing Magaz. 2008, 25(1): 116-129.

[4] A. Hassanien and A. S. Vorobyov, Phased-MIMO Radar: A tradeoff between Phased-Array and MIMO Radars. IEEE Trans on Antennas and Propagation, 2010, 58(6): 3137-3151.

[5] D. R. Fuhrmann, J. P. Browning, and M.Rangaswamy, Signaling Stratefies Design for Hybrid MIMO Phasedarray Radar. IEEE Journal, Selected Topics in Signal Processing, 2010, 4(1): 66-78.

[6] A. Deligiannis, S. Lambotharan. Beamforming for Fully-Overlapped Two-Dimensional Phased-MIMO Radar, IEEE Conference, 2015: 0599-0604.

[7] Chenglong Zhu, Hui Chen, and Huaizong Shao. Joing phased-MIMO and Nested-Array Beamforming for Increased Degrees-of-Freedom. Hindawi Publishing Corporation, International Journal of Antennas and Propagation, 2015.

[8] P. Pal and P. P. Vaidyanathan. Nested Arrays inTwo Dimensions, Part I: Geometrical Considerations. IEEE Trans on Signal processing, 2012, 60(9): 4694-4705.

[9] P. Pal and P. P. Vaidyanathan. Nested Arrays inTwo Dimensions, Part II: Application in Two Dimensional Array Processing. IEEE Trans on Signal processing , 2012, 60(9): 4706-4718.

[10] S. Q. Liu, B. H. Wang, L. J. Li, X. Li, S. Cao. DOA estimation method of Two-Dimensional Hybrid MIMO Phased-array Radar. Journal of Xidian University. 2017,(03):169-175. 